\title{
Quantitative Measurement of Local Infrared Absorption and Dielectric Function with Tip-Enhanced Near-Field Microscopy
}

\author{
Alexander A. Govyadinov, ${ }^{\dagger}$ Iban Amenabar, ${ }^{\dagger}$ Florian Huth, ${ }^{\dagger,}$ P. Scott Carney, ${ }^{\text {I }}$ \\ and Rainer Hillenbrand ${ }^{*},, \S$ \\ ${ }^{\dagger}$ CIC nanoGUNE Consolider, 20018 Donostia-San Sebastián, Spain \\ ${ }^{+}$Neaspec GmbH, 82152 Martinsried, Germany \\ ${ }^{\text {II } D e p a r t m e n t ~ o f ~ E l e c t r i c a l ~ a n d ~ C o m p u t e r ~ E n g i n e e r i n g ~ a n d ~ t h e ~ B e c k m a n ~ I n s t i t u t e ~ f o r ~ A d v a n c e d ~ S c i e n c e ~ a n d ~ T e c h n o l o g y, ~ U n i v e r s i t y ~ o f ~}$ \\ Illinois, Urbana, Illinois 61801, United States \\ ${ }^{\S}$ IKERBASQUE, Basque Foundation for Science, 48011 Bilbao, Spain
}

\section{Supporting Information}

\begin{abstract}
Scattering-type scanning near-field optical microscopy (s-SNOM) and Fourier transform infrared nanospectroscopy (nano-FTIR) are emerging tools for nanoscale chemical material identification. Here, we push s-SNOM and nano-FTIR one important step further by enabling them to quantitatively measure local dielectric constants and infrared absorption. Our technique is based on an analytical model, which allows for a simple inversion of the near-field scattering problem. It yields the dielectric permittivity and absorption of samples with 2 orders of magnitude improved spatial resolution compared to far-field measurements and is applicable to a large class of samples including polymers and biological matter. We verify the capabilities by determining the local dielectric permittivity of a PMMA film from nano-FTIR measurements, which is in excellent agreement with far-field ellipsometric data. We further obtain local infrared absorption spectra with unprecedented accuracy in peak position and shape, which is the key to quantitative chemometrics on the nanometer scale.
\end{abstract}

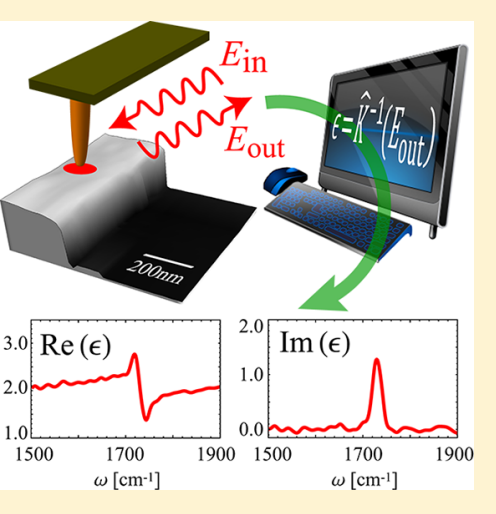

SECTION: Spectroscopy, Photochemistry, and Excited States

$\mathrm{R}$ adiation in the infrared (IR) and terahertz (THz) spectral regions is sensitive to molecular and electronic properties of matter, which makes it an excellent instrument for nondestructive identification and characterization of materials. Fourier transform infrared spectroscopy (FTIR) is thus a powerful and widely used technique for chemical materials identification based on IR fingerprint absorption spectroscopy. ${ }^{1}$ Likewise, IR ellipsometry ${ }^{2}$ or impedance spectroscopy ${ }^{3}$ can be utilized to measure the dielectric permittivity of a sample. Together, these techniques provide a complete identification and characterization of optical properties of a sample. However, the spatial resolution in both techniques is limited by diffraction to about half of the illumination wavelength $\lambda$, limiting their application potential in chemistry, biology, nanoscience, and the semiconductor industry, where the examination of single nano-objects is often desired.

The diffraction limit can be circumvented by scattering-type near-field optical microscopy (s-SNOM), which provides wavelength-independent nanoscale resolution even at IR and $\mathrm{THz}$ frequencies. ${ }^{4} \mathrm{~s}-\mathrm{SNOM}$ is a scanning probe technique often based on atomic force microscopy (AFM). In addition to the sample topography, s-SNOM yields optical amplitude and phase images, obtained by interferometric detection of the radiation scattered from the illuminated probe (typically a sharp metalized AFM tip). The scattered radiation depends on the near-field interaction between the tip and the sample, thus providing access to the optical properties of the sample at the nanoscale (we further refer to such properties as local)..$^{5-15}$

By employing broad-band illumination and performing Fourier transform spectroscopy of the tip-scattered light, sSNOM enables local spectroscopy (nano-FTIR) of plasmons and phonons, ${ }^{16-19}$ (bio)minerals, ${ }^{20}$ and polymers ${ }^{21,22}$ with less than $20 \mathrm{~nm}$ spatial resolution. It has been further shown that the local chemical identity of a polymer can be obtained by direct comparison of nano-FTIR spectral features with conventional FTIR spectra. ${ }^{21}$ While being successful in such spectroscopic material identification, quantitative measurements of the local permittivity of a sample, analogously to what is achieved with far-field ellipsometry or impedance spectroscopy, has so far proven elusive in s-SNOM and nanoFTIR.

Here, we present a technique for the quantitative determination of the local dielectric permittivity from sSNOM data, which provides 2 orders of magnitude improvement in spatial resolution compared to far-field measurements. The key to this achievement is a theoretical treatment that

Received: February 28, 2013

Accepted: April 1, 2013

Published: April 1, 2013 
allows for an analytical inversion of the near-field scattering problem. The technique is applicable to important material classes (which we refer to as weak oscillators), such as polymers and organic matter, and is suitable for processing s-SNOM data obtained by single-frequency or broad-band (nano-FTIR) measurements. We demonstrate the capabilities of our approach by determining the frequency-dependent local dielectric permittivity of a PMMA film from nano-FTIR measurements, which shows an excellent agreement with the dielectric permittivity obtained by far-field ellipsometry.

Our approach is based on the standard theoretical description of s-SNOM, where the amplitude- and phaseresolved measurement of the scattered field is described by the scattering coefficient $\sigma=E_{\mathrm{s}} / E_{\mathrm{i}}$ that relates the field $E_{\mathrm{s}}$ scattered by the tip to the field $E_{\mathrm{i}}$ incident upon it. ${ }^{4}$ The field at the tip location can be written as $\left(1+r_{\mathrm{s}}\right) E_{\mathrm{j}}$, where the reflection coefficient $r_{\mathrm{s}}$ accounts for the reflection of the field at the sample surface (see Figure 1). This field polarizes the tip,

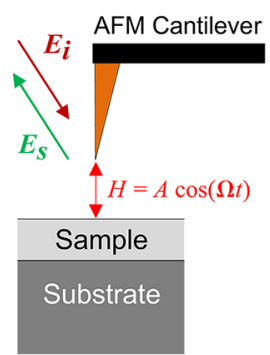

(a)

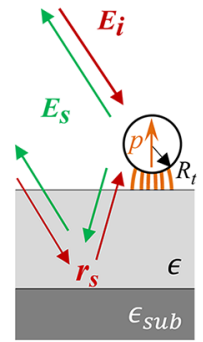

(b)

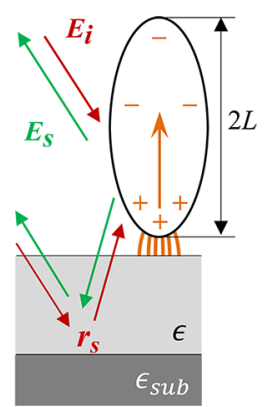

(c)
Figure 1. (a) s-SNOM schematics: An oscillating AFM tip is illuminated with an IR beam, and the backscattering is detected. (b) Point-dipole model: The tip is regarded as a point dipole $p$, which is acquired due to external illumination and near-field interaction with the sample. (c) Finite-dipole model: The tip is modeled as a long conductive spheroid with $L \gg R_{\mathrm{t}}$, which results in a large separation of induced charges. Only the charges in the tip apex participate in the near-field interaction with the sample.

yielding an effective dipole moment $p=\alpha_{\text {eff }}\left(1+r_{\mathrm{s}}\right) E_{\mathrm{i}}$, where $\alpha_{\text {eff }}$ is the effective polarizability of the tip that accounts for the near-field interaction between the tip and the sample. By reciprocity, the backscattered radiation of this dipole in the far field is $E_{\mathrm{s}}=\left(1+r_{\mathrm{s}}\right) p$, that is, the dipole backscatters directly and via reflection at the sample surface. ${ }^{4,23,24}$ The scattering coefficient can thus be written as

$$
\sigma=\alpha_{\text {eff }}\left(1+r_{\mathrm{s}}\right)^{2}
$$

Note that the scattering coefficient is complex-valued, as the tip-sample interaction can produce a phase difference between $E_{\mathrm{s}}$ and $E_{\mathrm{i}}$.

There exist two major analytic models that describe $\alpha_{\text {eff. }}$ The point-dipole model $^{4}$ (PDM, Figure $1 \mathrm{~b}$ ) regards the tip as a small sphere of radius $R_{\mathrm{t}}$ and polarizability $\alpha_{0}=4 \pi\left(\varepsilon_{\mathrm{t}}-1\right) /\left(\varepsilon_{\mathrm{t}}\right.$ $+2) R_{\mathrm{t}}^{3}$, with $\varepsilon_{\mathrm{t}}$ being the permittivity of the tip material. The quasi-static solution of the scattering problem yields the effective polarizability

$$
\alpha_{\text {eff }}=\alpha_{0}[1-f(H) \beta(\varepsilon)]^{-1}
$$

where $f(H)=\alpha_{0} /\left(16 \pi\left(R_{\mathrm{t}}+H\right)^{3}\right)$ is a function of the tip height $H$ above the sample surface, and $\beta=(\varepsilon-1) /(\varepsilon+1)$ is the quasi-static reflection coefficient that depends only on the sample permittivity $\varepsilon$.

The more sophisticated finite-dipole model (FDM, Figure 1c) takes into account the elongated shape of the tip and models it within electrostatic approximation as a conductive spheroid. ${ }^{24}$ In FDM, the effective polarizability can be written as

$$
\alpha_{\text {eff }}=C\left(1+\frac{1}{2} \frac{f_{0}(H) \beta(\varepsilon)}{1-f(H) \beta(\varepsilon)}\right)
$$

where the (height-independent) constant $C$ and heightdependent functions $f_{0}(H)$ and $f(H)$ are defined by the tip dimensions and material, particularly by the tip apex radius $R_{t}$ and an effective tip length $L$ that is almost invariable among standard AFM tips (expressions for $C, f_{0}$, and $f$ are given in the Supporting Information).

Equations 2 and 3 can be solved for $\beta$, and thus, $\varepsilon$ can be found. However, the value of $\alpha_{\text {eff }}$ required for such inversion is not directly accessible in s-SNOM because the scattering coefficient $\sigma$ measured in experiments is dominated by background scattering. To suppress the unavoidable background and to extract the near-field interaction, the tip is oscillated vertically with a small amplitude of the same order of magnitude as the tip radius $A \sim R_{\mathrm{t}} \ll \lambda$, at a frequency $\Omega$ of a few hundred $\mathrm{kHz}$ (see Figure 1a). The detector signal (which is proportional to the $E_{\mathrm{s}}$ due to interferometric detection) is then demodulated at higher harmonics $n \Omega$ of the oscillation frequency, ${ }^{6,25}$ with $n \geq 2$. The demodulation of the interferometric detector signal can be described by the complex Fourier transform of $\sigma(H)$ with respect to time $t$, as the height depends on time, $H=H(t)$, due to the vertical oscillation of the tip. ${ }^{26}$ The $n$th Fourier coefficient

$$
\sigma_{n}(\beta)=\hat{F}_{n}[\sigma(\beta, H(t))]=\int \sigma(\beta, H(t)) \exp (\mathrm{i} n \Omega t) \mathrm{d} t
$$

is proportional to the $n$ th-order demodulated detector signal.

In case the sample dielectric permittivity is known, $\sigma_{n}$ can be calculated and compared with s-SNOM data, which allows for an excellent quantitative description of measured data. ${ }^{12,17-19,24,27}$ However for an unknown sample, the extraction of $\varepsilon$ presents a nontrivial theoretical problem ${ }^{28-30}$ partially due to an essentially nonalgebraic relation between $\sigma_{n}$ and $\varepsilon$ in eq 4. Below, we develop an analytic solution for this problem that is valid for samples with $|\beta| \lesssim 1$ (we used $\lesssim$ to show that the actual limitation is $|f \beta|<1$, which allows $\beta$ to be slightly larger than 1), which includes weak molecular oscillators $^{2}$ (polymers, biological matter).

In eqs 2 and 3 describing the tip-sample near-field interaction, $\alpha_{\text {eff }}$ depends on the sample dielectric permittivity only through $\beta(\varepsilon)$. Furthermore, $\beta$ only appears in products with (model-dependent) functions of the tip height $H$

$$
\alpha_{\text {eff }}=\alpha_{\text {eff }}(f(H) \beta(\varepsilon))
$$

In this case, Taylor expansion of $\alpha_{\text {eff }}$ in eqs 2 and 3 in powers of $f \beta$ yields

$$
\alpha_{\text {eff }}(f \beta)=\sum_{j=0}^{\infty} \alpha^{(j)}(f \beta)^{j}
$$

where $\alpha^{(j)}$ are the expansion coefficients. The height-dependent function $f(H)$ in the expansion is less than unity for most s- 
SNOM experiments (see Figure S1, Supporting Information). This improves the convergence of eq 6 and extends its applicability to materials whose $\beta$ is slightly larger than 1 , such as metals and doped semiconductors (apart from regions close to plasma or phonon resonance where $|f \beta|$ can reach unity and the expansion 6 diverges).

Equation 6 makes $\alpha_{\text {eff }}$ separable in regards to the tip height $H$ and sample permittivity $\varepsilon$ (contained in $\beta$ ). The demodulation procedure, eq 4 , affects only height-dependent functions $f$ and $\alpha^{(j)}$, resulting in $\sigma_{n}$ also being separable. The latter for $n \geq 1$ is

$$
\sigma_{n}=\left(1+r_{\mathrm{s}}\right)^{2} \sum_{j=1}^{\infty} \hat{F}_{n}\left[\alpha^{(j)} f^{j}\right] \beta^{j}
$$

Equation 7 constitutes the core for determining the local permittivity from s-SNOM data. Truncated at a particular order $J$, it represents a simple polynomial equation that connects $\sigma_{n}$ to $\beta$. The coefficients in front of $\beta^{j}$ do not depend on sample permittivity and can be computed. If $\sigma_{n}$ is known, eq 10 can then be solved for $\beta$ analytically or via numerical routines that are robust and widely available. The permittivity $\varepsilon$ is subsequently found from $\beta$ according to

$$
\varepsilon=\frac{1+\beta}{1-\beta}
$$

The accuracy of the resulting solution can be controlled by the expansion order $J$.

A typical s-SNOM experiment, however, does not directly yield $\sigma_{n}$ but measures the quantity $T \sigma_{n}$, where $T$ is the response function of the setup. $T$ includes, among other things, the detector responsivity. To eliminate $T$, the detector signal is normalized to a reference signal obtained on a sample with well-known optical properties. This yields the complex-valued near-field contrast $\eta_{n}$

$$
\eta_{n}=\frac{\sigma_{n}}{\sigma_{n, \text { ref }}}
$$

At demodulation orders of $n>2$ and sufficiently small tip oscillation amplitudes $A$, background contributions are typically fully suppressed, ${ }^{4,6,10,25}$ yielding a pure near-field contrast. We note that due to the complex-valued normalization, the argument of $\eta_{n}$ yields the relative phase between the nearfield signal of the sample compared to the near-field signal of the substrate.

For samples that constitute an optically thin film (thickness $\Delta \ll \lambda$ ) deposited on a thick substrate (see Figure 1), the farfield reflection coefficient $r_{\mathrm{s}}$ is almost unaffected by the film presence (see Figure S3, Supporting Information). By taking the reference measurement on the substrate, we obtain

$$
\eta_{n}=\sum_{j=1}^{J} \frac{\hat{F}_{n}\left[\alpha^{(j)} f^{j}\right]}{\hat{F}_{n}\left[\alpha_{\text {eff,ref }}\right]} \beta^{j}
$$

where $\alpha_{\text {eff,ref }}$ is the effective polarizability that corresponds to the reference measurements. Because the permittivity of the reference is known, $\alpha_{\text {effref }}$ does not have to be expanded but can be calculated exactly (within the validity of the tip-sample interaction model). This in principle allows for treatment of near-field contrasts with any substrate.

In the simplest case of $J=1$, eq 10 simplifies to

$$
\eta_{n} \approx \frac{\beta}{\beta_{\text {ref }}}
$$

from which one immediately recovers the fundamentals of nano-FTIR absorption spectroscopy. ${ }^{21}$ Indeed, eq 11 states that in the first approximation, s-SNOM measures the quasi-static reflection coefficient $\beta . \beta$ is analogous to Fresnel reflection coefficients in electrodynamics that are typically purely real and acquire imaginary parts (and, therefore, phase) at frequencies near absorption lines in the sample's spectrum. According to eq 11, $\operatorname{Im}\left(\eta_{n}\right) \propto \operatorname{Im} \beta$, and thus, the $\operatorname{Im}\left[\eta_{n}(\omega)\right]$ in nano-FTIR matches well with the FTIR absorption spectra that directly relate to the absorption coefficient $\kappa(\omega)=\operatorname{Im}\left(\varepsilon(\omega)^{1 / 2}\right) \propto$ $\operatorname{Im}[\beta(\omega)]$. We can conclude that $\operatorname{Im}\left[\eta_{n}(\omega)\right]$ yields the local absorption of the sample and thus have defined $a_{n}(\omega)=$ $\operatorname{Im}\left[\eta_{n}(\omega)\right]$ as nano-FTIR absorption. ${ }^{21}$

Note that relation 9 does not depend on a particular model (i.e., is model-free) because all model-dependent functions $f$ and $\alpha^{(j)}$ have canceled out in eq 11. We also emphasize that to perform material identification according to far-field FTIR databases, one has to measure $a_{n}$ rather than the phase $\phi_{n}=$ $\operatorname{Arg}\left(\eta_{n}\right)$ as previously suggested. ${ }^{22,31}$ As we demonstrate below, $a_{n}$ represents a better approximation for $\kappa$ than $\phi_{n}$.

To compare different near- and far-field quantities, we performed nano-FTIR measurements on a $200 \mathrm{~nm}$ thick poly(methyl methacrylate) (PMMA) film deposited on a $\mathrm{CaF}_{2}$ substrate. To this end, we employed a commercial s-SNOM (Neaspec $\mathrm{GmbH}$ ) and a standard Au-coated AFM tip (apex radius $R_{\mathrm{t}} \approx 30 \mathrm{~nm}$ ) oscillating at $\Omega=115 \mathrm{kHz}$ and with a 50 $\mathrm{nm}$ amplitude. The tip was illuminated by a coherent broadband mid-infrared beam from a difference frequency generator $^{32}$ with beam parameters and the configuration of the illumination similar to those in ref 21 . The backscattered light was analyzed with an asymmetric Fourier transform spectrometer, providing complex-valued near-field spectra. The spectra of PMMA were obtained less than $200 \mathrm{~nm}$ from the film edge and have been normalized to those measured on $\mathrm{CaF}_{2}$ (see Figure 2a,b), yielding the local complex-valued nearfield contrast (Figure 2c,d).

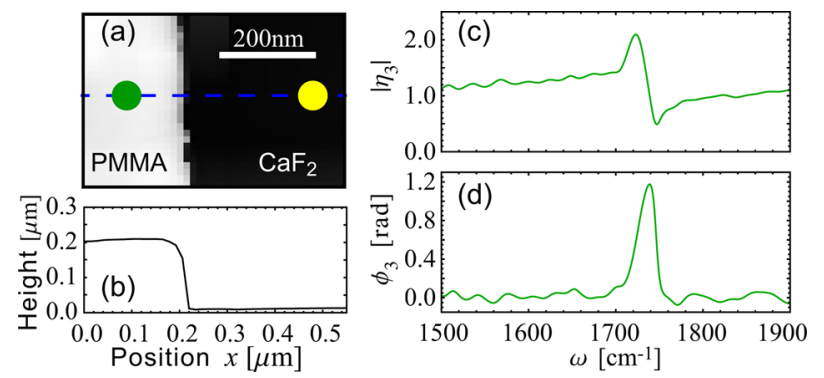

Figure 2. Sample topography (a) and its profile (b) taken along the dashed line. Green and yellow dots mark the locations where, respectively, the PMMA and reference measurements have been performed. The corresponding amplitude (c) and phase (d) spectra of the near-field contrast between PMMA and $\mathrm{CaF}_{2}$ measured at the third harmonic. The spectra were measured with a spectral resolution of $16.7 \mathrm{~cm}^{-1}$ and smoothed using bandwidth-limited interpolation (see the spectral resolution and nano-FTIR calibration in the Supporting Information).

In Figure 3, we compare the nano-FTIR absorption $a_{3}(\omega)$ and phase $\phi_{3}(\omega)$ with the absorption coefficient $\kappa(\omega)$ obtained by conventional FTIR spectroscopy. In all spectra, the resonance peak corresponding to the stretching of the $\mathrm{C}=\mathrm{O}$ bond of PMMA is clearly seen. The peak position in $a_{n}(\omega)$ agrees well (within $2 \mathrm{~cm}^{-1}$ ) with the peak in $\kappa(\omega)$, while the 


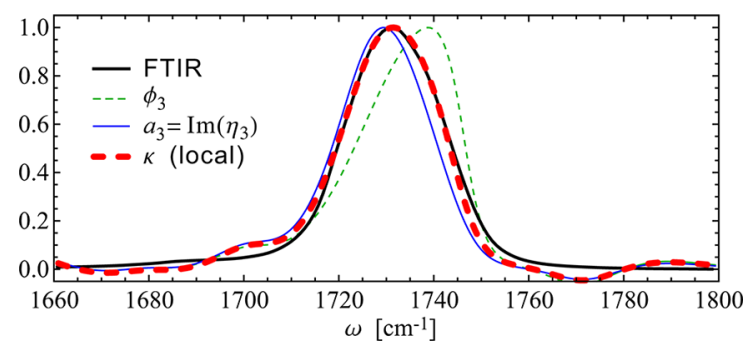

Figure 3. Spectra of the nano-FTIR absorption $a_{3}$ (thin blue) and phase $\phi_{3}$ (thin dashed green) of a $200 \mathrm{~nm}$ thick PMMA film. Note the good agreement between the position of the $a_{3}$ peak and the PMMA's molecular absorption line ( $\mathrm{C}=\mathrm{O}$ stretching) obtained via conventional FTIR (thick black). The local absorption coefficient $\kappa$ computed from the local dielectric permittivity obtained by inversion of eq 10 is plotted in thick dashed red. Note a near-perfect match with the FTIR spectrum. All curves are normalized to their maxima. Only the region around the absorption peak is shown to highlight the differences between the curves.

peak in the phase $\phi_{3}(\omega)$ spectrum is shifted by about $7 \mathrm{~cm}^{-1}$, which could lead to the misidentification of materials.

We now invert eq 11 to find the first-order approximation for $\beta$ and determine $\varepsilon$ according to eq 8 . For the inversion, we have used the permittivity of $\varepsilon_{\mathrm{CaF}}$ from ref 33 . The result is depicted in Figure 4. The derived values of $\varepsilon$ differ by up to an order of

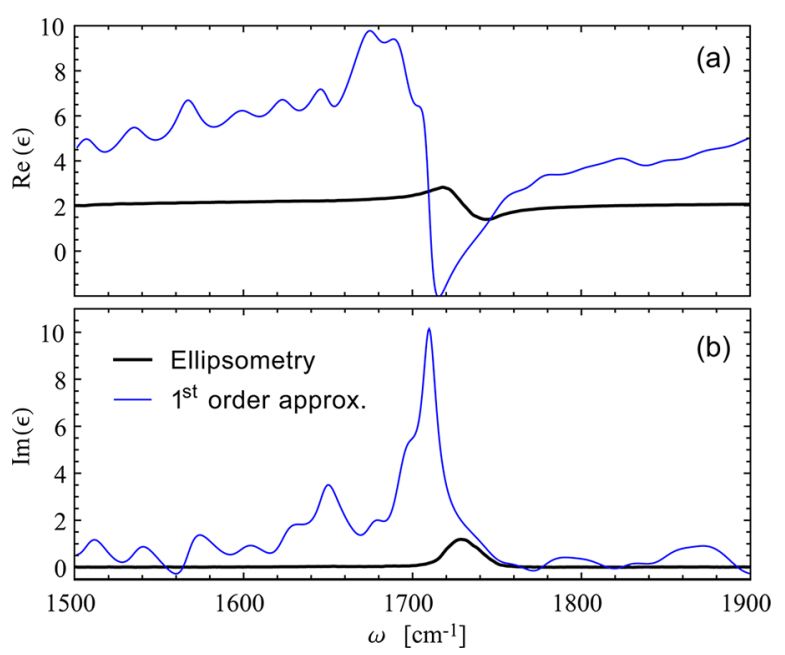

Figure 4. An approximation to the real (a) and imaginary (b) parts of $\varepsilon$, keeping only the first-order term in the Taylor expansion of $\alpha_{\text {eff }}$. The necessity of higher-order corrections for the quantitative analysis is apparent.

magnitude from the reference ellipsometric measurements. ${ }^{34}$ This demonstrates that the first-order approximation $J=1$ is inappropriate for the quantitative determination of the local sample permittivity. Additionally, $\beta=\beta_{\text {ref }} \eta_{n}$ (and thus the obtained $\varepsilon$ ) depends on the demodulation order $n$ (and, in fact, the tip oscillation amplitude) due to such dependence of the near-field contrast. $6,35,36$

For determining $\varepsilon$ quantitatively, we seek a more accurate solution for $\beta$ from eq 10 . To that end, we increase the expansion order $J$ until the change in the obtained permittivity from one expansion order to another is less than a desired accuracy (in the following, $J=9$, and the corresponding relative error in permittivity is $<5 \times 10^{-5}$; see Figure S2, Supporting Information). Figure 5 displays the permittivity of PMMA

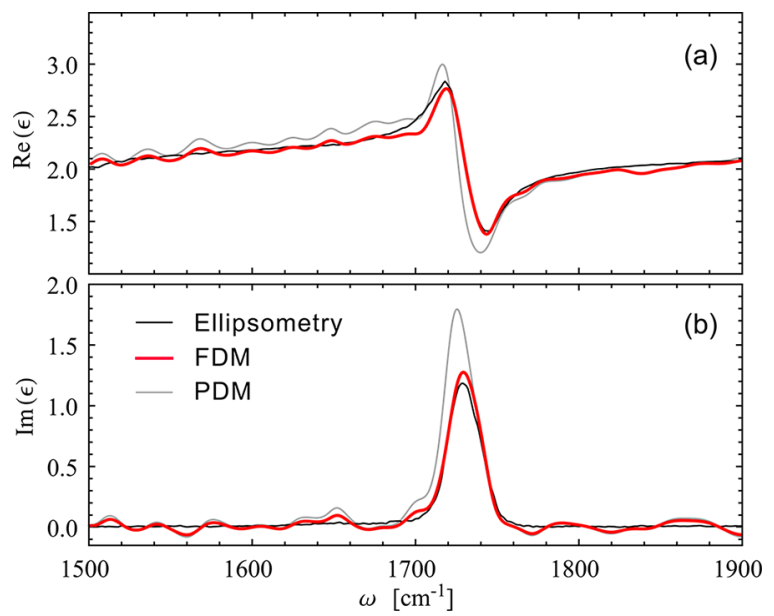

Figure 5. Real (a) and imaginary (b) parts of the local dielectric permittivity of the PMMA film obtained though the inversion procedure described in the text using FDM (thick red) and PDM (thin gray). The thin black line corresponds to the far-field ellipsometric data obtained from a $5 \mu \mathrm{m}$ PMMA film.

inferred using the PDM and FDM. We observe an excellent agreement between the FDM result and the dielectric permittivity of PMMA derived from ellipsometry. The FDM model parameters are $L=600 \mathrm{~nm},{ }^{17} A=50 \mathrm{~nm}$, and $R_{\mathrm{t}}=30$ $\mathrm{nm}$ (a brief description of model parameters and the corresponding expressions for computing permittivity can be found in the Supporting Information). We note, however, that the result is almost insensitive to the variation of these parameters. The inversion based on PDM yields a less accurate permittivity.

The local absorption coefficient $\kappa(\omega)$ (dashed red curve in Figure 3) can now be computed from the permittivity obtained from nano-FTIR measurements. We find that not only the position of the resonance peak but also the shape of the spectral line is now in excellent agreement with the far-field measurements.

In eq 10, we have neglected the modification of far-field reflection from that of the bare substrate due to film presence. The far-field reflection can be accounted for perturbatively. One first finds $\varepsilon$ according to the procedure shown above, which allows for computing the $r_{\mathrm{s}}$ (for a particular film thickness). The inversion is then repeated with $r_{\mathrm{s}}$ retained in eq 10. We have performed such refinement for our film (with thickness obtained from the sample topography, Figure $2 b$ ) and found that the correction in $\varepsilon$ around resonance is $\leq 5 \%$, while off-resonance, it is well below $1 \%$ (see Figure $\mathrm{S} 4$ in the Supporting Information).

The results presented here employ two widely used models of tip-sample interaction in which the film is assumed to be transversely infinitely extended. Because the field localization around the tip apex is typically on the order of the tip radius, ${ }^{10,37}$ we expect our approach to apply well to nanoobjects that are larger than this size. The sensitivity to the vertical composition of the film, however, can extend to the depths of several tip radii, ${ }^{23,38-40}$ with corresponding limitation on the smallest thickness of studied nano-objects. In the employed experimental setup, objects with dimensions $\gtrsim 200$ $\mathrm{nm}$ can be investigated, which we estimated from the invariance of the PMMA spectrum beginning $100 \mathrm{~nm}$ away from the film edge. $^{21}$ Further reduction of object sizes can be performed by 
employing sharper tips $^{38}$ and smaller tip oscillation amplitudes. $^{36}$

In summary, we have presented a technique for extracting the local complex-valued dielectric permittivity and the absorption coefficient of a thin film from s-SNOM images and nano-FTIR spectra. Our approach is based on the direct inversion of the near-field scattering problem and does not require a model for the sample permittivity, which is typically associated with computationally intense and noise-sensitive minimization procedures. It directly returns the real and imaginary parts of permittivity without relying on Kramer's-Kronig relations ${ }^{41}$ and thus allows for the determination of complex-valued dielectric permittivity from single-frequency measurements. Our work lays the foundation for quantitative optical imaging and spectroscopy of materials on the nanometer scale, which opens new frontiers for chemometrics, materials and biosciences.

\section{ASSOCIATED CONTENT}

\section{S Supporting Information}

The description of the nano-FTIR measurements and calibration, details for the tip-sample models and their parameters, and the supporting Figures S1-S4, showing the dependence of functions $f$ on the tip height, the maximum relative error in the inversion result as a function of the expansion order $J$, the influence of the PMMA film on the farfield reflection from the sample and the resulting relative error in the obtained $\varepsilon$. This material is available free of charge via the Internet at http://pubs.acs.org.

\section{AUTHOR INFORMATION}

\section{Corresponding Author}

*E-mail: r.hillenbrand@nanogune.eu.

\section{Notes}

The authors declare the following competing financial interest(s): R.H. is a co-founder of Neaspec $\mathrm{GmbH}$, a company producing scattering-type scanning near-field optical microscope systems, such as the one used in this study. All other authors declare no competing financial interests.

\section{ACKNOWLEDGMENTS}

We would like to thank Federico Golmar for the preparation of the PMMA sample, Andrey Chuvilin for verifying the tip radius with SEM, and Alexander Bittner for fruitful discussions. This research was funded by the ERC Starting Grant No. 258461 (TERATOMO) and the Etortek-2011 project (nanoiker) of the Department of Industry of the Basque Government.

\section{REFERENCES}

(1) Griffiths, P. R.; de Haseth, J. A. Fourier Transform Infrared Spectrometry; Wiley: New York, 2007.

(2) Roeseler, A. Infrared Spectroscopic Ellipsometry; Akademie-Verlag: Berlin, 1990.

(3) Inzelt, G.; Lang, G. G. Electropolymerization; Wiley-VCH Verlag GmbH \& Co. KGaA: Weinheim, Germany, 2010; pp 51-76.

(4) Keilmann, F.; Hillenbrand, R. In Nano-Optics and Near-Field Optical Microscopy; Richards, D., Zayats, A., Eds.; Artech House: Boston/London, 2009.

(5) Knoll, B.; Keilmann, F. Near-Field Probing of Vibrational Absorption for Chemical Microscopy. Nature 1999, 399, 134-137.

(6) Hillenbrand, R.; Keilmann, F. Complex Optical Constants on A Subwavelength Scale. Phys. Rev. Lett. 2000, 85, 3029-3032.
(7) Hillenbrand, R.; Taubner, T.; Keilmann, F. Phonon-Enhanced Light Matter Interaction at The Nanometre Scale. Nature 2002, 418, $159-162$.

(8) Brehm, M.; Taubner, T.; Hillenbrand, R; Keilmann, F. Infrared Spectroscopic Mapping of Single Nanoparticles and Viruses at Nanoscale Resolution. Nano Lett. 2006, 6, 1307-1310.

(9) Qazilbash, M. M.; Brehm, M.; Chae, B.-G.; Ho, P.-C.; Andreev, G. O.; Kim, B.-J.; Yun, S. J.; Balatsky, A. V.; Maple, M. B.; Keilmann, F.; Kim, H.-T.; Basov, D. N. Mott Transition in $\mathrm{VO}_{2}$ Revealed By Infrared Spectroscopy and Nano-Imaging. Science 2007, 318, 17501753.

(10) Huber, A. J.; Keilmann, F.; Wittborn, J.; Aizpurua, J.; Hillenbrand, R. Terahertz Near-Field Nanoscopy of Mobile Carriers in Single Semiconductor Nanodevices. Nano Lett. 2008, 8, 37663770.

(11) Jacob, R.; Winnerl, S.; Fehrenbacher, M.; Bhattacharyya, J.; Schneider, H.; Wenzel, M. T.; Ribbeck, H.-G. V.; Eng, L. M.; Atkinson, P.; Schmidt, O. G.; Helm, M. Intersublevel Spectroscopy on Single InAs-Quantum Dots by Terahertz Near-Field Microscopy. Nano Lett. 2012, 12, 4336-4340.

(12) Stiegler, J. M.; Huber, A. J.; Diedenhofen, S. L.; Rivas, J. G.; Algra, R. E.; Bakkers, E. P. A. M.; Hillenbrand, R. Nanoscale FreeCarrier Profiling of Individual Semiconductor Nanowires by Infrared Near-Field Nanoscopy. Nano Lett. 2010, 10, 1387-1392.

(13) Kopf, I.; Grunwald, C.; Brundermann, E.; Casalis, L.; Scoles, G.; Havenith, M. Detection of Hybridization on Nanografted Oligonucleotides Using Scanning Near-Field Infrared Microscopy. J. Phys. Chem. C 2010, 114, 1306-1311.

(14) Stiegler, J. M.; Abate, Y.; Cvitkovic, A.; Romanyuk, Y. E.; Huber, A. J.; Leone, S. R.; Hillenbrand, R. Nanoscale Infrared Absorption Spectroscopy of Individual Nanoparticles Enabled by Scattering-Type Near-Field Microscopy. ACS Nano 2011, 5, 6494-6499.

(15) Paulite, M.; Fakhraai, Z.; Li, I. T. S.; Gunari, N.; Tanur, A. E.; Walker, G. C. Imaging Secondary Structure of Individual Amyloid Fibrils of a $\beta 2$-Microglobulin Fragment Using Near-Field Infrared Spectroscopy. J. Am. Chem. Soc. 2011, 133, 7376-7383.

(16) Amarie, S.; Ganz, T.; Keilmann, F. Mid-infrared Near-Field Spectroscopy. Opt. Express 2009, 17, 21794-21801.

(17) Amarie, S.; Keilmann, F. Broadband-Infrared Assessment of Phonon Resonance in Scattering-Type Near-Field Microscopy. Phys. Rev. B 2011, 83, 045404.

(18) Huth, F.; Schnell, M.; Wittborn, J.; Ocelic, N.; Hillenbrand, R. Infrared-Spectroscopic Nanoimaging with a Thermal Source. Nat. Mater. 2011, 10, 352-356.

(19) Zhang, L. M.; Andreev, G. O.; Fei, Z.; McLeod, A. S.; Dominguez, G.; Thiemens, M.; Castro-Neto, A. H.; Basov, D. N.; Fogler, M. M. Near-Field Spectroscopy of Silicon Dioxide Thin Films. Phys. Rev. B 2012, 85, 075419.

(20) Amarie, S.; Zaslansky, P.; Kajihara, Y.; Griesshaber, E.; Schmahl, W. W.; Keilmann, F. Nano- FTIR Chemical Mapping of Minerals in Biological Materials. Beilstein J. Nanotechnol. 2012, 3, 312-323.

(21) Huth, F.; Govyadinov, A. A.; Amarie, S.; Nuansing, W.; Keilmann, F.; Hillenbrand, R. Nano- FTIR Absorption Spectroscopy of Molecular Fingerprints at $20 \mathrm{~nm}$ Spatial Resolution. Nano Lett. 2012, 12, 3973-3978.

(22) Xu, X. G.; Rang, M.; Craig, I. M.; Raschke, M. B. Pushing the Sample-Size Limit of Infrared Vibrational Nanospectroscopy: From Monolayer toward Single Molecule Sensitivity. J. Phys. Chem. Lett. 2012, 3, 1836-1841.

(23) Aizpurua, J.; Taubner, T.; Garcia de Abajo, F. J.; Brehm, M.; Hillenbrand, R. Substrate-enhanced infrared near-field spectroscopy. Opt. Expr. 2008, 16, 1529-1545.

(24) Cvitkovic, A.; Ocelic, N.; Hillenbrand, R. Analytical Model for Quantitative Prediction of Material Contrasts in Scattering-Type NearField Optical Microscopy. Opt. Express 2007, 15, 8550-8565.

(25) Labardi, M.; Patane, S.; Allegrini, M. Artifact-Free Near-Field Optical Imaging by Apertureless Microscopy. Appl. Phys. Lett. 2000, $77,621-623$. 
(26) Note that $\sigma$ is still represented in the frequency domain, that is, $\sigma=\sigma(\omega)$, as implied by its prior definition. Due to the vertical tip oscillation, it also acquires the time dependence, $\sigma=\sigma(\omega ; t)$. Such a mixing of frequency and time domains is possible because the tip oscillation is 9 orders of magnitude slower compared to the IR frequency, and the tip can thus be regarded as stationary at any moment of time.

(27) Hauer, B.; Engelhardt, A. P.; Taubner, T. Quasi-Analytical Model for Scattering Infrared Near-Field Microscopy on Layered Systems. Opt. Express 2012, 20, 13173-13188.

(28) Greffet, J.; Carminati, R. Image Formation in Near-Field Optics. Prog. Surf. Sci. 1997, 56, 133-237.

(29) Carney, P.; Schotland, J. C. Near-Field Tomography. Inside Out: Inverse Problems; Mathematical Sciences Research Institute: Berkeley, CA, 2003; Vol. 47, pp 133-168.

(30) Gaikovich, K. Subsurface Near-Field Scanning Tomography. Phys. Rev. Lett. 2007, 98, 183902.

(31) Taubner, T.; Hillenbrand, R.; Keilmann, F. Nanoscale Polymer Recognition by Spectral Signature in Scattering Infrared Near-Field Microscopy. Appl. Phys. Lett. 2004, 85, 5064-5066.

(32) Keilmann, F.; Amarie, S. Mid-Infrared Frequency Comb Spanning an Octave Based on an Er Fiber Laser and DifferenceFrequency Generation. J. Infrared, Millimeter, Terahertz Waves 2012, 33, 479-484.

(33) Bezuidenhout, D. In Handbook of Optical Constants of Solids; Palik, E. D., Ed.; Academic Press: Burlington, MA, 1997; pp 815 835.

(34) The FTIR data is adopted from ref 21, and the IR ellipsometry data for PMMA is courtesy of A. Roseler, which has been used in ref 27.

(35) Taubner, T.; Hillenbrand, R.; Keilmann, F. Performance of Visible and Mid-Infrared Scattering-Type Near-Field Optical Microscopes. J. Microsc. 2003, 210, 311-314.

(36) Taubner, T.; Keilmann, F. Effect of Tip Modulation on Image Contrast in Scattering-Type Near-Field Optical Microscopy. J. Korean Phys. Soc. 2005, 47, 213-216.

(37) Esteban, R.; Vogelgesang, R.; Kern, K. Full Simulations of the Apertureless Scanning Near Field Optical Microscopy Signal: Achievable Resolution and Contrast. Opt. Express 2009, 17, 25182529.

(38) Raschke, M. B.; Lienau, C. Apertureless Near-Field Optical Microscopy: Tip-Sample Coupling in Elastic Light Scattering. Appl. Phys. Lett. 2003, 83, 5089-5091.

(39) Taubner, T.; Keilmann, F.; Hillenbrand, R. Nanoscale-resolved subsurface imaging by scattering-type near-field optical microscopy. Opt. Express 2005, 13, 8893-8899.

(40) Krutokhvostov, R.; Govyadinov, A. A.; Stiegler, J. M.; Huth, F.; Chuvilin, A.; Carney, P. S.; Hillenbrand, R. Enhanced resolution in subsurface near-field optical microscopy. Opt. Express 2011, 20, 593600.

(41) Aspnes, D. In Handbook of Optical Constants of Solids; Palik, E. D., Ed.; Academic Press: Boston, MA, 1985. 